\title{
Potentiometric Study on the First Ionization of Carbonic Acid in Aqueous Solutions of Sodium Chloride
}

\author{
REINO N ÄSÄNEN
}

Propaedeutic-Chemical Labonatory of the University of Helsinki, Finland

\begin{abstract}
Tn a recent investigation by the author on barium carbonate ${ }^{1}$ the values of Ithe ionization constants of carbonic acid in sodium chloride solutions were required for the numerical treatment of the solubility equilibrium. This led the author to study the ionization of carbonic acid in sodium chloride solutions. The experiments for determination of the first ionization constant are described in this paper. The results on the second ionization were published earlier ${ }^{2}$.
\end{abstract}

The ionization equilibrium in carbonic acid solution is given by

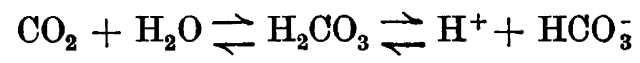

The first ionization constant is defined by

$$
\frac{\left[\mathrm{H}^{+}\right]\left[\mathrm{HCO}_{3}^{-}\right]}{\left[\mathrm{CO}_{2}\right]}=K_{1}
$$

and the carbon dioxide molality is given by

$$
\left[\mathrm{CO}_{2}\right]=S p_{\mathrm{CO}_{3}}
$$

where $p_{\text {co2 }}$ is the partial pressure of carbon dioxide and $S$ the Henry's law constant. The elimination of $\left[\mathrm{CO}_{2}\right]$ from equations (2) and (3) leads to the expression

$$
\left[\mathrm{HCO}_{3}^{-}\right]=\frac{K_{1} S p_{\mathrm{co}_{\mathrm{a}}}}{\left[\mathrm{H}^{+}\right]}
$$


To obtain the buffer capacity in the usual manner a small quantity of sodium hydroxide is added and then titrated with hydrochloric acid. Near the inflection point the following relation exists

$$
y=c_{A}-c_{B}=\left[\mathrm{H}^{+}\right]-\left[\mathrm{HCO}_{3}^{-}\right]
$$

where $c_{A}$ denotes the concentration of hydrochloric acid and $c_{B}$ that of sodium hydroxide. From (4) and (5) is obtained

$$
y=c_{A}-c_{B}=\left[\mathrm{H}^{+}\right]-K_{1} S p_{\mathrm{co}_{2}} /\left[\mathrm{H}^{+}\right]
$$

The first derivative of $y$ with respect to $p \mathrm{H}$ is

$$
P=d y / d p \mathrm{H}=2.303 \quad\left\{\left[\mathrm{H}^{+}\right]+K_{1} S p_{\mathrm{Co}_{\mathbf{2}}} /\left[\mathrm{H}^{+}\right]\right\}
$$

When the second derivative of $y$ is put equal to zero it follows that at inflection point

$$
\left[\mathrm{H}^{+}\right]=\sqrt{K_{1} S p_{\mathrm{CO}_{2}}}
$$

Introducing this equation into equation (7) we obtain for buffer capacity at inflection point the expression

$$
P=4.606 \sqrt{K_{1} S p_{\mathrm{CO}_{2}}}
$$

Solving this equation for $K_{1}$ the result is

$$
K_{1}=\frac{4.714 \cdot 10^{-2} P^{2}}{S p_{\mathrm{co}}}
$$

By means of equation (10) when the quantity $P$ and the constant $S$ are known, it is possible to calculate the ionization constant $K_{1}$. Equation (9) represents a very simple individual form of the general equation of the buffer eapacity at inflection point ${ }^{3}$.

\section{EXPERIMENTAL}

The temperature of the water bath in which the measurements were carried out was kept constant with an accuracy of $\pm 0.01^{\circ}$. A hydrogen electrode was used as indicator electrode. The titration vessel used was similar to that described earlier by the 
present author ${ }^{1}$. The gas mixture entering the titration vessel from a tank filled with tank hydrogen and carbon dioxide contained $16.60 \%$ carbon dioxide by volume. The hydrochloric acid solution used in the determination of the buffer capacity was $\mathbf{0 . 0 1 9 2 0}$ $N$. Kahlbaum's chemicals for analysis were used. The potential jumps were measured with a Leeds \& Northrup $\mathrm{K}$ type potentiometer.

For further experimental details the reader is referred to the earlier papers of the author ${ }^{4}$.

\section{RESULTS}

Table 1 includes the results of the measurements. The ionic strength $\mu$ in this table is defined by $\mu=\frac{1}{2} \Sigma m_{i} z_{i}^{2}$, where $m_{i}$ denotes molality of ion indicated. The buffer capacity $P$ of carbonic acid solution for, in other words, the reciprocal of the maximum slope of the titration curve) was calculated from the potentiometric data with the aid of equations 4

and

$$
P=2.303 \Delta a C R T / \Delta E F V m_{\mathrm{o}}
$$

$\Delta E=\Delta m+\frac{1}{6}\left\{\left(\Delta m-\Delta_{1}\right)+\left(\Delta m-\Delta_{2}\right)\right\}-\frac{\left(\Delta m-\Delta_{1}\right)\left(\Delta m-\Delta_{2}\right)}{2\left\{\left(\Delta m-\Delta_{1}\right)+\left(\Delta m-\Delta_{2}\right)\right\}}$

In these equations the denotations are:

$C$ the normality of hydrochloric acid solution,

$\Delta a$ the small increment of hydrochloric acid solution in $\mathrm{ml}$,

$R$ the gas constant,

$T$ the absolute temperature,

F Faraday charge,

$m_{\mathrm{o}}$ the amount of water in the titrated solution at the inflection point in grams,

$\Delta_{1}, \Delta m, \Delta_{2}$ successive changes of potential produced by the addition of $\Delta a \mathrm{ml}$ of hydrochloric acid solution ( $\Delta m$ is the maximum change).

The partial pressure $p_{\mathrm{co} 2}$ of carbon dioxide expressed in atm was calculated from barometric pressure, the composition of the gas mixture $(16.60 \%$ carbon dioxide) and the vapor pressure of water. The vapor pressure of the sodium chloride solution was obtained from "International Critical Tablesi) ${ }^{5}$. The Henry's law constant $S$ is obtained from a recent paper of Harned and Davis ${ }^{6}$. 
Table 1. The calculation of the first ionization constant of carbonic acid in sodium chloride solutions.

\begin{tabular}{|c|c|c|c|c|c|}
\hline$t$ & $\mu$ & $P \cdot 10^{4}$ & $p_{\mathrm{CO} 2}$ & $S \cdot 10^{2}$ & $p K_{1}$ \\
\hline 5 & 0.00312 & 2.800 & 0.1633 & 6.40 & 6.4506 \\
\hline 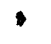 & 0.0142 & 2.939 & 0.1645 & 6.39 & 6.4120 \\
\hline$\bullet$ & 0.143 & 3.448 & 0.1648 & 6.16 & 6.2584 \\
\hline " & 1.065 & 3.830 & 0.1631 & 4.85 & 6.0584 \\
\hline 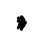 & 3.135 & 2.774 & 0.1619 & 3.10 & 6.1410 \\
\hline 15 & 0.00312 & 2.607 & 0.1633 & 4.56 & 6.3662 \\
\hline$"$ & 0.0142 & 2.786 & 0.1640 & 4.55 & 6.3095 \\
\hline$"$ & 0.143 & 3.256 & 0.1643 & 4.40 & 6.1600 \\
\hline$"$ & 1.065 & 3.641 & 0.1645 & 3.50 & 5.9646 \\
\hline 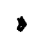 & 3.135 & 2.625 & 0.1608 & 2.31 & 6.0547 \\
\hline 25 & 0.00312 & 2.477 & 0.1631 & 3.44 & 6.2878 \\
\hline$"$ & 0.0142 & 2.588 & 0.1620 & 3.43 & 6.2442 \\
\hline$"$ & 0.143 & 3.065 & 0.1621 & 3.32 & 6.0851 \\
\hline$"$ & 1.065 & 3.369 & 0.1618 & 2.69 & 5.9105 \\
\hline 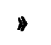 & 3.135 & 2.525 & 0.1622 & 1.81 & 5.9889 \\
\hline 35 & 0.00312 & 2.236 & 0.1582 & 2.68 & 6.2542 \\
\hline$"$ & 0.0142 & 2.354 & 0.1577 & 2.67 & 6.2075 \\
\hline$"$ & 0.143 & 2.816 & 0.1574 & 2.59 & 6.0322 \\
\hline$"$ & 1.065 & 3.056 & 0.1550 & 2.13 & 5.8762 \\
\hline$"$ & 3.135 & 2.320 & 0.1591 & 1.45 & 5.9574 \\
\hline 45 & 0.00312 & 2.011 & 0.1491 & 2.18 & 6.2317 \\
\hline$\|$ & 0.0142 & 2.120 & 0.1492 & 2.19 & 6.1861 \\
\hline$"$ & 0.143 & 2.530 & 0.1490 & 2.12 & 6.0199 \\
\hline - & 1.065 & 2.798 & 0.1500 & 1.73 & 5.8477 \\
\hline$"$ & 3.135 & 2.112 & 0.1520 & 1.18 & 5.9322 \\
\hline
\end{tabular}

The thermodynamic ionization constant is calculated with the aid of equation

$$
p K_{1}=p K_{1,0}-\frac{2 A \sqrt{\mu}}{1+\sqrt{\mu}}+B \mu+D \mu^{2}
$$

in which the constant $A$ is calculated from

$$
A=0.4883+0.75545 \cdot 10^{-3} t+0.1743 \cdot 10^{-5} t^{2}+0.11665 \cdot 10^{-7} t^{3}
$$

obtained by Scatchard 7 on the basis of the recent tables of Birge ${ }^{8}$. The values of $p K_{1,0}, B$ and $D$, evaluated by the method of least squares, are compiled in table 2. With these values $p K_{1}$ may be computed with a maximum deviation of 0.0107 and average deviation of 0.0036 from the $p K_{1}$-values in table 1 . The values of the thermodynamic ionization constant may be calculated from

$$
p K_{1^{\prime} 0}=6.5720-0.012173 t+0.00013329 t^{2}
$$


Table 2. The thermodynamic first ionization constant of carbonic acid and the parameters of equation (13).

\begin{tabular}{rcccc}
\multicolumn{1}{c}{$t$} & $p K_{1,0}$ & $2 A$ & $B$ & $D$ \\
5 & 6.5140 & 0.9842 & 0.02401 & 0.0183 \\
15 & 6.4210 & 1.000 & 0.03037 & 0.0183 \\
25 & 6.3489 & 1.017 & 0.06524 & 0.00861 \\
35 & 6.3104 & 1.035 & 0.07782 & 0.00671 \\
45 & 6.2939 & 1.054 & 0.07676 & 0.00716
\end{tabular}

The maximum and average deviation of the values calculated by equation (15) from those in table 2 is 0.0021 and 0.0012 respectively. The constants $B$ and $D$ may be calculated satisfactorily by

and

$$
B=0.01660+0.01530 t
$$

$$
D=0.02028-0.000339 t
$$

\section{DISCUSSION}

The values of the thermodynamic constant obtained in this investigation agree excellently with the recent results of Harned and Bonner ${ }^{9}$. The maximum and average deviation of $p K_{1},{ }_{0}$-values of this paper from those of Harned and Bonner is 0.0048 and 0.0026 respectively. In sodium chloride solutions, however, the maximum and average deviation of $p K_{1}$-values increases as the ionic strength rises, being at $1 M$ solution about 0.04 and 0.02 respectively. The values of this paper are smaller than the values of Harned and Bonner.

The greatest error in the method used in the present investigation arises from the measurement of the potential jumps. An accuracy better than $0.5 \%$ is available. This causes an error of less than $1 \%$ in the ionization constant. The other errors are of the same order of magnitude as in the method used by Harned and Bonner.

The free energy, heat content, heat capacity and entropy of ionization may be derived in the usual manner from the ionization constants obtained above. These calculations are not performed in this connection because it is the purpose of the author to complete the measurements in the future.

\section{SUMMARY}

The"buffer capacity of carbonic acid solutions with added sodium chloride has been determined up to a concentration of $3 M$ salt and at temperatures of $5^{\circ}, 15^{\circ}, 25^{\circ}, 35^{\circ}$ and $45^{\circ}$. 
From these potentiometric data and from the solubility of carbon dioxide in these solution the first ionization constant of carbonic acid has been calculated over the above range of concentration and temperature.

Equations for computing the ionization constant at temperatures from $5^{\circ}$ to $45^{\circ}$ and at ionic strength from 0 to $3 M$ are given.

Equation for computing of the thermodynamic first ionization constant as function of temperature is given.

\section{REFERENCES}

1. Näsänen, R. Ann. Acad. Sci. Fennicae A II Chemica 17 (1946).

2. Näsänen, R. Suomen Kemistilehti 19 B (1946) 89.

3. Cf. Kilpi, S. Z. physik. Chem. (A) 175 (1935) 239.

4. Näsänen, R. Z. physik. Chem. (A) 188 (1941) 272, (A) 190 (1942) 183, (A) 191 (1942) 54; Suomen Kemistilehti 16 B (1943) 1, 17 B (1944) 11, 18 B (1945) 45.

5. International Critical Tables, New York (1929) p. 370.

6. Harned, H. S., and Davis, R. Jr. J. Am. Chem. Soc. 65 (1943) 2030.

7. Scatchard, G. J. Am. Chem. Soc. 65 (1943) 1249.

8. Birge, R. T. Rev. Mod. Phys. 13 (1941) 233.

9. Harned, H. S., and Bonner, F. T. J. Am. Chem. Soc. 67 (1945) 1026. 